\title{
ROADSIDE HAZARD SIMULATION MODEL ITS DEVELOPMENT AND APPLICATION
}

\author{
By Hirotaka KOIKE*
}

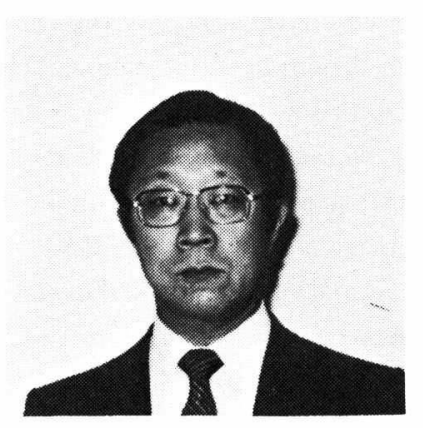

\section{INTRODUCTION}

The roadside is an integral part of any highway, and its design and maintenance must seek to satisfy a number of often conflicting requirements. It should, for example, be harmless to encroaching vehicles, pleasing in appearance, economical to build and economical and easy to maintain; it must be capable of accommodating traffic control and information signs, lighting standards, the pole-lines of telephone and power utilities. It must provide adequate drainage for the highway and it must provide space for the stopping of vehicles off the travelled portion of the highway. The requirement that the roadside be rendered harmless to encroaching vehicles is the specific focus of this paper.

A typical roadside contains many features that are potentially hazardous to encroaching vehicles and their occupants. The hazardous features may be objects, such as utility and highway lighting poles, sign posts, bridge abutments and trees, or the features may be parts of the more permanent roadside environment, such as ditches with front or back slopes and rock-cuts.

In most cases there is interaction among the hazardous features in the form of shielding effects, where one object or set of objects may mask the hazardous effects of other objects or features. For example, trees lining a rock-cut or a river will effectively render these features harmless; however if the trees are removed both features will become more prominent hazards. Whether, and by how much, the degree of hazard changes when shielding is altered will depend on the characteristics and location of the features involved. If, in the above example, the trees are substantial and located fairly close to the road they may represent more of a hazard than the features they are shielding. If they are located close to the rock-cut and river which are far away from the road, however, their removal may not materially change the hazard

* Professor, Department of Civil Engineering, the University of Utsunomiya 
level.

Vehicles encroaching on the roadside do so at varying speeds and along paths at different angles to the travelled portion of the road. Under otherwise identical circumstances different speeds and directions of encroachment will result in different consequences for an encroaching vehicle and its occupants. For example, at small angles of encroachment features and objects located some distance away from the travelled portion of the road will generally not constitute hazards since an encroaching vehicle, generally, will be unable to reach them.

The consequences of vehicle interactions, collisions, with objects and other roadside features depend on many factors such as vehicle speed, mass and rigidity; object dimensions, mass and strength; the number of vehicle occupants, their physical condition and age and whether or not occupant restraint systems are used.

Little is known about how many vehicles are encroaching on the roadside. According to the Hutchinson and Kennedy's study of over 6 million vehicle trips during the three-and-one-half years period, less than $1 / 5000$ of $1 \%$ involved a vehicle encroachment upon the median (Hutchinson and Kennedy 1966). This low rate of encroachment makes it difficult to collect data on roadside accidents.

There are a number of earlier studies on the subject of roadside hazards. Many of them deal with specific roadside features. Among these features are; guardrails (Michie 1971, 1972, Ross and Post 1973, Ross 1976, Glennon and Tamburri 1977); utility poles (Edward 1969, Walton 1973, Graf 1976); median dikes (Dunlap and Grote 1972); shoulder design (Dean 1976); and crash cushions (Hirsh 1970, Marquis 1976). Other studies deal with more than one kind of roadside hazards. They tend to be more statistical than analytical in their methodology (Tutt and Nixon 1970, Glennon 1974, OECD Road Research Group 1975, Wright and Robertson 1976, Nowak 1976).

In estimating the consequences of roadside encroachments, two basic approaches have been used. One approach, based on an extremely detailed three-dimensional trajectory estimation using computer simulation, is represented by the Highway-Vehicle-Object Simulation Model (HVOSM) developed by Calspan over a decade since 1966 (McHenry 1967, 1968, Segal 1976). HVOSM was used to simulate the interaction between a vehicle and other objects or terrain features, such as : cable barriers (Pinkney et al. 1972) ; concrete median barriers (Young et al. 1973, Labra 1977) ; sloping culvert grates (Ross and Post 1972, 1974, ). HVOSM is designed for the detailed examination of a single vehicle trajectory and not for the general evaluation of roadside effects and its use for such a purpose is prohibitively expensive even with current high speed computer technology.

The other approach is to construct various models based on the notion of severity indices. One of the best known among these models is the one developed by Glennon and published in the AASHTO Traffic Barrier Guide (AASHTO 1977). The problem with these models is that they all assume one average departure angle (11 degrees in Glennon's model and 15 or 20 degrees in the De Leuw Cather model) and departure speed variation is not adequately accounted for.

Using a constant departure angle forces the model to assume one possible departure point as defined by the position of an object. Any additional obstacles located within the "shadow" of protection so defined behind the primary object or guardrail must be assumed immune from collision. In fact this is far from the truth, as about $60 \%$ of departure angles are less than 11 degrees for near-side encroachments. In addition, the severity indices employed are usually based on some form of accident statistic ratio (injury accidents/total accidents) which is arrived at by aggregating accident information, often from a wide range of sources in which obstacle categories have been combined out of necessity or expediency. Severity indices will be discussed in detail in the next section.

The need for a new approach which can bridge these two extremes was called for in order to estimate the relative safety of a variety of roadside configurations and hazard characteristics. The Roadside Hazard Simulation Model (RHSM) was developed for Transport Canada in the late 1970's primarily as an efficient 
tool for comparing various roadside designs and obstacles over a wide range of encroachment conditions.

\section{MEASURES OF SEVERITY}

The RHSM requires a measure of severity for off-the-road incidents as an essential system component. The so called "severity index" should have the following characteristics:

- It must be a single value unique to incident consequences.

- It must be conceptually appealing and consistent among various kinds of incidents.

- It must be easy to compute from experimental or actual accident records.

- It must be capable of translation into fatalities, injuries and property damage.

At least a dozen different "severity indices" have been used in various studies, and a brief review is in order to justify the development of a new index. It should be mentioned that the coverage of literature is by no means exhaustive or extensive. Conventional severity indices have been divided into two major groups; one is statistical in nature and the other is analytical, although the latter may require statistical treatment for its calibration.

In the statistical approach, the severity index is based on the number of accidents reported. The most common definition is as follows (see De leuw Cather 1975, Glennon 1973, Roy Jorgenson 1976, Hall 1976) :

$$
S I(\text { Severity Index })=\frac{F+I}{N}
$$

or in a modified form (Glennon 1977) :

$$
S I=\frac{25 F+6 I+P}{N}
$$

where : $F=$ Number of fatal accidents,

$I=$ Number of injury accidents,

$P=$ Number of accidents with property damage only,

$N=$ Total number of accidents

The difference between (1) and (2) is the weighting in the second formula by psuedo-cost-conversion factors. The problem with these statistical severity indices is that they depend on database quality and do not necessarily yield consistent results.

The analytical approach is based on some of the physical characteristics of accidents. Among these are vehicle damage, velocity change and deceleration, and the level of injury or probability of fatality for the occupants. The latter, the severity level for the occpants of a vehicle, is a secondary factor which is derived from the consequences of vehicle motion ; it may be regarded as a function of the severity index related to the vehicle itself.

However, the wide range of occupant characteristics such as age, use of restraint system and relative position in the vehicle compartment makes it rather difficult to establish relationships between the vehicle severity index and that for the occupants. An example of such an effort is found in the study by Watson and Shiels (1975) where the nine-point American Medical Association's Abbreviated Injury Scale (AIS) level is estimated from the age of the occupants and the Collision Deformation Classification (CDC) numbers.

There are many other severity indices related to vehicle occupants' tolerance limits, but little has been done to establish the relationships between vehicle and occupant severity indices. The study done at the University of Saskatchewan is one example (1976).

The severity indices associated with the vehicle itself are also numerous. Some of them are given below.

The severity index based on deceleration and occupant tolerance limit was used in some studies of impacts with guardrails and other roadside structures (Olson 1974, Weaver 1975, Ross 1972). It compares average deceleration with the tolerance limit for each directional component.

$$
S I=\sqrt{\left(G_{x} / G_{x m}\right)^{2}+\left(G_{y} / G_{y m}\right)^{2}+\left(G_{z} / G_{z m}\right)^{2}}
$$

where: $G_{x}, G_{y}, G_{z}=$ Deceleration in the $x, y$ and $z$ axes, 
$G_{x m}, G_{y m}, G_{z m}=$ Maximum tolerable deceleration in the three axes

The maximum tolerable deceleration vary with the restraint system used, and also among different studies (Graham 1967).

A severity index based on the change in velocity $(\Delta V)$ has gained wide popularity since the development of the SMAC and CRASH accident reconstruction programs made it possible to compute this quantity (McHenry 1973, 1976, Jones 1974). However, $\Delta V$ has the shortcoming of failing to indicate the abruptness of a crash, resulting in poor correlation with other severity parameters such as the AIS level (Burgett 1975). This inconsistency becomes more prominent when crashes with various roadside features with different stiffness is considered. Obviously the severity of a crash with a bridge abutment is not the same as for a collision into soft bushes, even though the changes of velocity might be identical.

The physical damage to a vehicle is another measure of severity. It is given by the absolute value of deformation of the vehicle body, in inches, as computed in Equivalent Barrier Speed (Campbell 1974), or by scaled indices such as CDC or TAD (Rouze 1969). Here again inconsistencies become apparent when these indices are used to describe single vehicle crashes with features of varying stiffness.

There is a gradual trend to consider more than one parameter associated with an accident. A good example is the work done by Tarriere and others in which two parameters are used to classify accidents, namely the speed change and the mean deceleration (Tarriere 1975). However, these parameters were not combined into a single measure.

A more desirable form of severity measure is one in which both change of speed and abruptness of change (deceleration) are combined into a single value. Moffatt proposed the following formula for a desirable severity measure (Moffatt 1976).

$S I=f(\Delta V, \Delta S)$

In this formula the severity index is a function of velocity change $(\Delta V)$ and an equivalent stopping distance $(\Delta S)$, which are the first and second integrals of the deceleration pulse during a crash.

A moving vehicle possesses a certain amount of kinetic energy which is dissipated when the vehicle comes to a standstill. Any reduction in the vehicle's speed, whether it is a crash with another vehicle or object, or a simple braking maneuver, dissipates kinetic energy.

If energy dissipation is accomplished over a reasonable period of time neither vehicle nor occupants suffer damage. The ordinary braking maneuver falls in this category. However, when the energy is dissipated in a short time, an accident is considered to have taken place. In an extreme case the energy dissipation is almost instantaneous, such as when crashing into a rigid barrier. This would be a severe accident. Accordingly, it is the time of energy dissipation that makes the difference in the consequences. The amount of energy dissipation per unit of time is defined as power and is given in the following formula :

$$
P=\frac{d}{d t} \text { (Kinetic Energy) }=\frac{d}{d t}\left(\frac{1}{2} m V^{2}\right)=m V \frac{d V}{d t} \text {. }
$$

where : $P=$ power, $V=$ velocity, $m=$ mass, $t=$ time

If $(5)$ is divided by the mass of the vehicle, a uniform severity measure is obtained which is independent of vehicle weight.

$$
\begin{aligned}
S I & =\frac{P}{m} \\
& =V \frac{d V}{d t}=V a \ldots \ldots \ldots \ldots \ldots \ldots \ldots \ldots \ldots \ldots \ldots \ldots \ldots \ldots \ldots \ldots \ldots \\
& =V \frac{d S}{d t} \frac{d V}{d S}=V^{2} \frac{d V}{d S} \text { or } V^{2} \frac{\Delta V}{\Delta S} \ldots \\
& =a t \frac{d V}{d t}=a^{2} t \ldots \ldots \ldots \ldots \ldots \ldots \ldots
\end{aligned}
$$

where : $\Delta V=$ velocity change, $\Delta V=$ distance travelled, $a=$ deceleration

Equation ( 7 ) satisfies the requirement established in (4) as one way of combining velocity change and 
stopping distance.

It may also be written in the form of equations (6) and (8). Equation (6) is one step further from Tarriere's study because it combines speed change and deceleration into a single value, power.

Equation ( 8 ) deserves some attention because of its similarity with Gadd's severity index (Gadd 1966) : $S I=t a^{2.5}$

In his formulation, a $S I=1000$ is considered as the human tolerance limit; it was later modified to 1500. Similar severity indices are proposed in other studies (e.g., Newman 1975), all of which are given as a function of time and deceleration. Although there is much criticism (Hodgson 1970, Versace 1971) of Gadd's log-linear approximation of very limited data and the appropriateness of the constants used, it is probably not coincidental that the severity index based on the power concept resembles Gadd's severity index. For the time range used in the formulation of the latter there is little justification for the exponent value of 2.5 as was pointed out by Versace. In fact, the curve based on ( 8 ) closely matches that of (9). It is not known whether the human tolerance limit is linearly related to power, but this fact does not invalidate the severity index based on the power concept as an adequate indicator of the level of severity associated with the vehicle itself.

The theoretical paper by DiLorenzo examined the correlation between power and bodily injury (DiLorenzo 1976). It should be noted that his paper is the first one which uses power as the primary factor in injury causation. It lends credibility to the use of power as a measure of accident severity. It should be noted that the power concept achieves the combination of more that one parameter in a logical way. It is also worthwhile to note that the interchangeability of equations (6) through ( 8 ) will be of help when some parameter values are missing.

\section{THE MODEL DEVELOPMENT}

Vehicles encroach roadsides at various angles and speeds, and from any point along the road. The consequences of these different initial conditions of encroachment may vary widely. It is desirable therefore to take every combination of initial speed, angle, and encroachment position into consideration. The overall effect of a roadside environment can be assessed only after all these possible incidents and their consequences are aggregated.

Due to the great number of possible combinations of roadside, object, vehicle and occupant characteristics a method is needed whereby the hazard level of any one of these combinations may be estimated quickly and economically, and with an accuracy comparable with that of the rest of the information which will be considered in deciding whether or not a particular roadside should be modified. A computer simulation model (HVOSM), capable of reproducing vehicle movements with a fair degree of accuracy, is already available (Segal 1976). However, the cost of making the computations required with this model would make any reasonably comprehensive examination of different roadside features prohibitively expensive even with advanced high speed computers.

This severe limitation of HVOSM made it necessary to develop a less complex model. A simpler model, based on a point mass representation of a vehicle, has been developed. It can simulate a single vehicle trajectory at a fraction of the cost of running HVOSM.

The approach taken in the model was to actually simulate the movement of a vehicle encroaching at a certain speed and angle from a point along the edge of the pavement and to determine the effects of terrain features and objects on this movement. The amount of interaction between the vehicle and the roadside features is measured by the power required to change the vehicle's velocity. This power is transformed into probabilities that certain consequences will result. The weighted sum of all possible combinations of consequence-probabilities represents, as an aggregated measure, the hazard level of a particular roadside.

\section{(1) Model Basics}


In the model a vehicle is represented as a point mass and objects are expressed in terms of high friction coefficients. It must be noted that the use of friction coefficients to represent objects extends the usual meaning of this concept. In general terms, a moving mass on a surface with friction coefficient $\mu$ decelerates at the rate:

$$
a=\mu g
$$

'To extend this concept, a greater deceleration can be achieved if the $\mu$ value is made larger. The extreme case is a crash to a rigid wall where the deceleration is extremely large, and so is the value of $\mu$ required to achieve this deceleration. Since the deceleration of a mass can assume any value on a continuous scale, the $\mu$ value will also follow a continuous scale. Moderate braking, encroachment to bush or a crash into a rigid rock-cut are all expressed by characteristic values of $\mu$. What particular value of $\mu$ is needed for particular features and conditions must be determined through model calibration. For instance, the $\mu$ value may vary with the speed of a vehicle as well as with the relative stiffness of objects in its way. A change of terrain slope can also become hazardous if the change is large enough to cause an abrupt increase in deceleration. This again is expressed by a high friction coefficient, at the point of velocity change. Based on this concept, a single vehicle trajectory model was developed.

\section{( 2 ) Terrain Adjustment}

RHSM develops a reasonable reproduction of a single vehicle trajectory, at a cost several orders of magnitude less than that of HVOSM. This submodel, which is called Point Mass System (PMS), treats a vehicle as a point with mass and computes acceleration, velocity and location at small increments of time. For some specific cases HVOSM was extensively used to calibrate PMS. Generally speaking, PMS yields results very close to those produced by HVOSM on flat terrain. However, on other than flat terrain correction factors must be introduced to account for airborne landing impacts and for dive-in impact that occur at the bottom of ditches or at the start of back slopes.

It is impossible to reproduce exactly the sprung mass movement of a vehicle simulated in HVOSM by using PMS. What is required in the PMS is the reproduction of high peaks in the power profile, and for this purpose PMS gives satisfactory results. The terrain adjustment factors are obtained by comparing HVOSM and PMS for a series of different terrains. When the terrain slope change is convex the point mass trajectory becomes airborne and then lands with an impact. In the PMS routine the landing impact is accounted for by the deceleration caused by an artificially high friction coefficient at the time of landing. A concave slope change will cause high power dissipation at the point of change; this is also accounted for by a high friction coefficient at the point of slope change.

The method of obtaining the adjustment factor for airborne cases is described as follows. HVOSM was used to simulate movements of a vehicle starting on flat terrain and landing on different down-grade slopes at different speeds and peak power values were generated. Regression analysis was used on 16 cases to derive the relationship between speed, slope angle and the ratio of maximum power to nominal power (the power developed at the point of landing in the adsence of impact). This power ratio, named the terrain adjustment factor, was used to determine the friction coefficient necessary to reproduce the power generated by the impact. A routine for calculating the equivalent friction coefficient values for landing impacts forms part of the simulation model.

$$
\begin{gathered}
F=0.8662-0.1852 V \tan A+0.256(V \tan A)^{2} . \\
(R \text { square }=0.957)
\end{gathered}
$$

where : $F=$ adjustment factor for friction coefficient,

$V=$ precrush speed $(\mathrm{m} / \mathrm{s})$,

$A=$ slope angle (degree)

The same calibration method was applied for concave slope changes. Nineteen experimental runs were made using HVOSM with the vehicle starting on flat terrain and running into different up-grade slopes at different speeds. The terrain adjustment factor for the friction coefficient for dive-in impacts is given as 
follows :

$F=0.8637+0.4961 V \tan A+0.07288(V \tan A)^{2}$

$(R$ square $=0.978)$

\section{(3) Object Adjustment}

Conceptually, objects on the roadside are defined as natural or man-made items. However, terrain geometry is excluded from this category. Both objects and terrain features can become hazards if they can be reached by a vehicle travelling fast enough to generate adverse consequences. In other words, objects are harmless if they are not accessible, or they produce little damage to the vehicle on contact.

When there is more than one object on the roadside, one or more of them may be shielded by other objects. The RHSM is capable of dealing with shielding effects. The advantage of the variable speed/angle approach over the conventional fixed average speed and angle method is easily understood if one imagines a case where two objects are in line at the average encroachment angle. The conventional model will ignore the second object because it is shielded by the first one even if the second one is more hazardous.

Even if a vehicle does reach an object the level of hazard can vary widely, depending on the characteristics of the object and the speed of impact with it. The severest impact is expected when a vehicle crashes to a rigid wall at high speed. Here the danage is experienced through deformation of the vehicle; this deformation is also the crash distance. For the same velocity change $(\Delta V)$ the shorter the crash distance, the higher the deceleration and power. A frontal crash to a rigid barrier establishes the upper limit of hazard for a given crash speed. All other objects are considered as some proportion of the rigid barrier case. Three distinct object categories are identified, although it is not always possible to classify an object in a single category. They are rigid, deformable but non-traversable and breakable or traversable objects.

In the rigid object category, the barrier which has dimensions equal to or greater than the cross-section of a vehicle should be rated highest in crash severity. Smaller rigid object such as concrete poles or rigid guardrails will allow longer crash distances if the vehicle is considered as a uniform body. In a real case, however, a hard point such as engine block may cause the vehicle to react as a unit and result in a smaller difference between full barrier and small rigid object crashes. Also, it should be noted that intrusion into the occupant compartment is very much dependent upon the vehicle structure. Consideration of this is beyond the scope of this paper.

Five experimental HVOSM runs into a rigid full barrier resulted in a close relationship between the maximum power generated due to the crash and the precrash speeds. This relationship is included in the model to produce the object adjustment factor for rigid full barrier cases. Less rigid objects are given adjustment factors expressed as a fraction of that for a rigid barrier.

$$
P=13.0 \mathrm{~V}^{2}
$$

$$
(R \text { square }=1.00)
$$

where : $P=$ maximum power $(\mathrm{W} / \mathrm{kg})$,

$$
V=\text { precrush speed }(\mathrm{m} / \mathrm{s})
$$

Both deformable objects and breakable objects will generate less power than a rigid barrier because of longer crash distances, resulting in lower deceleration, or smaller velocity changes. A crash to a deformable object lasts until the vehicle stops, whereas a crash to breakable object may release the vehicle after the object has been broken. Many objects may have both characteristics depending on the energy applied at the time of crash. An inventory of various objects and their characteristics in relation to a rigid barrier must be established. This will be an ongoing process of updating and replacing assumptions as information on crashes to various objects becomes available.

The RHSM considers all roadside hazards in terms of equivalent friction coefficients. The location and dimension of the object must also be considered. The roadside area is covered by an imaginary grid consisting of $1 \mathrm{~m} \times 1 \mathrm{~m}$ squares as the basic module. Each square is assigned a unique $\mu$ value, which can be 
a genuine terrain surface friction coefficient or the equivalent high $\mu$ value which charaterizes an object. Since the $1 \mathrm{~m}$ square grid is the smallest spatial unit, any object smaller than this must be considered to occupy an entire square. This is not unreasonable because the single vehicle trajectory routine assumes that the entire vehicle mass is located at the vehicle's centre of gravity. Considering an average vehicle to be $2 \mathrm{~m}$ wide it will collide with any object located within a path of this width.

\section{(4) Initial Encroachment Condition}

The RHSM model requires location along the road, angle and speed of encroachment to be entered as initial conditions. Lack of information concerning the distributions of encroachment angles and speeds makes some arbitrary assumptions necessary. Although it is outdated, the most widely used source of information about encroachment angles is the study done by the University of Illinois (Hutchinson and Kennedy 1966). According to this source the cumulative proportion of vehicles encroaching at angles equal or greater than a given value forms a negative exponential distribution.

The speed distribution of encroaching vehicles is also virtually unknown. Until more reliable data becomes available. it must be assumed that the speed of encroaching vehicles has the same normal distribution as traffic on the highway, although the model can accept any distribution form. For modelling purposes the speed distribution is divided into equal ranges of speed, each with its own probability of occurrence. The product of the probabilities for particular angles and speeds gives the relative probability that an encroachment with these characteristics will occur.

The probability discussed here is important because the level of hazard, and the consequences of a terrain or object feature, is detemined not only by the power developed when a vehicle reaches a point, but also by the probability of access to that point. When comparing two points which have the same power levels, but different probabilities of being reached, it is natural to assume that the one with higher probability is more hazardous than the other.

\section{(5) Probability of Consequences}

The single trajectory model calculates the power level and the location of a vehicle at various points in time as its primary outputs. However, the power level is only an intermediate result and difficult to interpret by the user. It must be translated into some other index which is more appealing and easier to understand. The basic question in a real encroachment situation is what happens to the vehicle and its occupants. There are four types of consequences expected. They are no damage, property damage only (PDO) which may include very minor injury, injury to the occupants, and fatality. It is often difficult to classify an accident into one category or another, but it can be done theoretically.

For a given level of power, there are four probabilities assigned to these consequences. For example, very low power will produce almost $100 \%$ no damage or PDO incidents and no injury or fatal accidents. As the power level increases, the proportions of injury and fatal accidents gradually increase. At high power levels almost all incidents end up as severe injury or fatal accidents. Two things must be noted here. One is that the sum of the four consequence probabilities must always be equal to one. The other is that the consequences can be different even at the same level of power, depending on the level of restraint use by the occupants. Two extreme cases (fully restrained and unrestrained) have been examined and relationships have been established between power level and consequence probabilities. Since little is known about the relationship between power and its consequences, the relationships established are crude and must be subject to future elaborations and improvements.

To cover the entire range of power spectrum, barrier crash experiments have been chosen. As for the consequences, a study done at the University of Saskachewan attempted to relate the AIS level and the CDC extent number (University of Saskachewan 1976). A study done by Campbell establishes the relationship between speed and crash distance in rigid barrier crashes (Campbell 1974). When the results of these two studies are combined, it is possible to establish a relationship between consequences and power levels. The results are shown in Fig. 1 One is for unrestrained and one for fully restrained 
occupants. Although the Saskachewan study differentiated between the age groups of the occupants, age 40 has been chosen to represent the entire population. It must be noted that AIS levels 0 and 1 are grouped as PDO, and AIS levels 5 and 6 are grouped as fatal. There is a minor adjustment applied at very low levels of power to account for unreported incidents which are likely of no damage or minor PDO. This adjustment resulted in an increase in PDO and a reduction in injury at lower power levels.
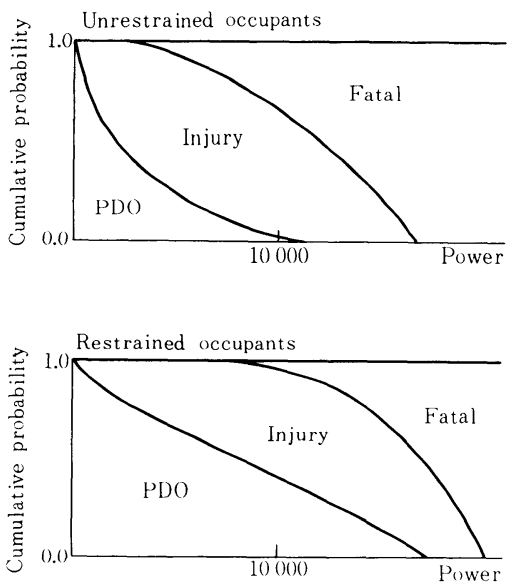

Fig. 1 Power and Consequence Probability.

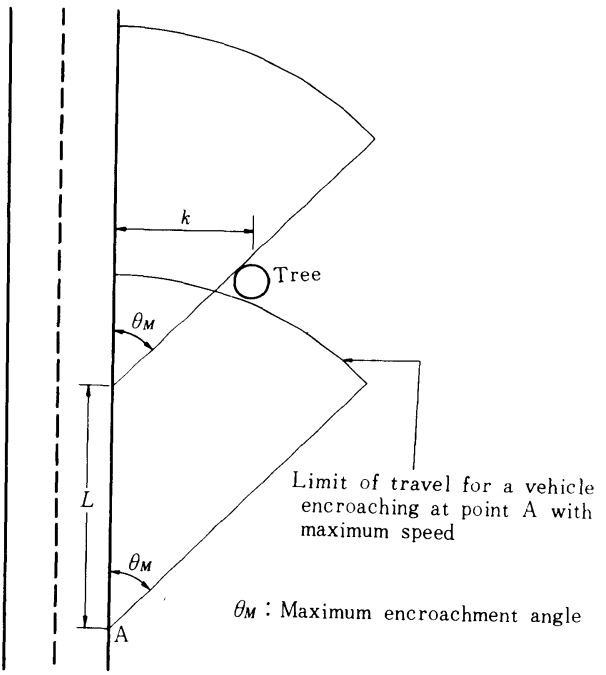

Fig. 2 Determining the Effect of a Tree.

\section{(6) Aggregation of Consequences}

Single vehicle trajectories simulated according to initial angle and speed conditions are aggregated in the grid matrix. When a vehicle encroaches at a point along the road, its trajectory is calculated using the PMS routine and the power developed at specified coordinate points is determined. The distance between two consecutive points is determined by the time increment used. The smaller the time increment, the more accurate is the trajectory. However the iteration time also increases. A good compromise between accuracy and efficiency is obtained when a trajectory has at least one point in each grid square. If more than one point falls within the same square for a particular trajectory, an average power value is computed and assigned. An exception to this rule is when power changes drastically within a square, such as where a landing impact or a crash to an object occurs. In such cases, the highest power is assigned to the square. The power calculated is then converted to consequences using the conversion tables discussed in the previous section. Each trajectory result is accumulated in the grid matrix until all trajectories have been considered.

So far the discussion has been concerned with but a single encroachment point. A fan-shaped trajectory sweep generated from other points, maybe only a few meters away from the point in consideration, may result in quite different power surfaces. A gradual shift of the encroachment point will result in overlapping, fan-shaped power surfaces (Fig. 2). Their combined consequences are obtained by aggregation parallel to the road over a certain distance, into a single set of values. The final result of the RHSM is the consequence probabilities of a vehicle encroaching a certain roadside section.

\section{SIMULATION EXAMPLES}

\section{(1) Object Relocation and Removal,}

A series of experiments have been made using hypothetical situations to demonstrate the performance of the model. This example demonstrates the interrelated effects of an isolated object with different terrain 
configurations. A rigid pole is located in two different positions on two different terrains, and generates a set of consequence probabilities. The terrain configurations are: (a) flat terrain and (b) standard trapezoidal ditch terrain (RCU 70) which is obtained from the Geometric Design Standards for Canadian Roads and Streets (metric edition) published by the Roads and Transportation Association of Canada. The same terrain surface friction coefficient $(0.7)$ was applied to both terrains. The locations of the rigid pole are at $4 \mathrm{~m}$ and $12 \mathrm{~m}$ from the edge respectively. Fig. 3 illusrtates the six alternatives examined in this experiment.

The speed distribution of encroaching vehicles is assumed to have a mean of $70 \mathrm{~km} / \mathrm{h}$ and a standard deviation of $10 \mathrm{~km} / \mathrm{h}$. Full brake application throughout the encroachment is also assumed. The results are summarized in Table 1. Three levels of seat belt usage are examined. Although the probabilities of fatal and injury accidents are very small in comparison with PDO or no damage for the section of road which includes the isolated rigid pole, the output indicates that the magnitudes of the consequences are in the right rank order. Some findings are as follows:

- The standard ditch terrain produces higher fatal and injury accident probabilities than does flat terrain.

- Fatal accidents probabilities decrease as the object is moved away from the edge of pavement and then removed in both terrains.

- Injury accident probabilities do not change as drastically as those for fatal accidents. This may be due to the fact that no differentiation is made within the injury accident category. In other words, the relative proportions of minor and serious injury accidents may be different although their combined probability remains nearly constant.

- The level of restraint system usage makes considerable difference in consequences. Full use of restraint systems reduces fatal and injury accident probabilities substantially.

- The differences in probabilities among the three object locations, including the no object case, indicate the effect of object removal and relocation. The same kind of treatment for a different terrain resulted in a different amount of probability changes. This indicates that effect of an object must be considered in relation to the terrain on which that object is located, and the effect of an object alone can not be isolated as a probability which is constant for any terrain. This finding is at variance with the conventional method of dealing with objects, where they are considered in isolation without regard to the terrain in which they are located.

\section{(2) Installation of Guardrail}

The next experiment conducted was an examination of the effects of a guardrail installed to protect an isolated rigid pole located $4 \mathrm{~m}$ from the edge of pavement. This is similar to the last alternative in the previous example of standard ditch terrain, and all input conditions are set to the same values as before. Two different lengths of guardrail are compared with no-guardrail condition. Fig. 4 shows the three basic configrations of objects (pole and guardrail). The rigidity of the guardrail in comparison to the rigid wall is arbitrarily assumed to be $80 \%$ for the end and $50 \%$ for the mid section. The two lengths are also chosen arbitrarily to give the effects of different lengths of guardrail. The result is shown in Table 2 where the unprotected case is repeated from the previous table. The findings are :

- The shorter guardrail has a lower fatal accident probability than the unprotected pole for all seat belt usage categories. Slightly lower injury accident probabilities are also indicated.

- The longer guardrail actually increases the hazard potential for most categories of restraint use except for the fully restrained fatal accident catagory.

Although this example is hypothetical, more rigorous examinations must be made with regard to the guardrail location and rigidity, it indicates that an excessively long guardrail can become more of a hazard than the object it is meant to shield. 


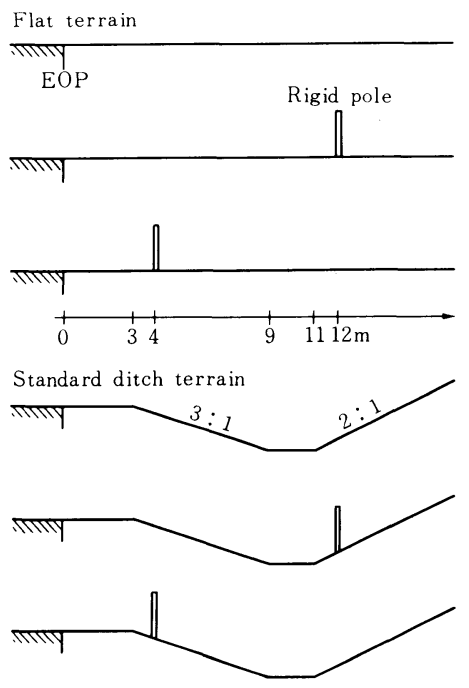

Fig. 3 Simulation Experiment (A) (Six alternative terrain/object configurations)

Table 1 Simulation Result (A) (Effects of relocation and removal of a rigid pole)

\begin{tabular}{|c|c|c|c|c|c|}
\hline \multirow{2}{*}{ Terrain } & \multirow{2}{*}{$\begin{array}{l}\text { Object } \\
\text { Location }\end{array}$} & \multirow{2}{*}{\begin{tabular}{|l|} 
Restraint \\
Usage \\
\end{tabular}} & \multicolumn{3}{|c|}{ Consequence probabilities } \\
\hline & & & $\mathrm{ND} / \mathrm{PDO}$ & Injury & Fatal \\
\hline \multirow[t]{3}{*}{ Flat } & No object & $\begin{array}{l}0 \% \\
100\end{array}$ & $\begin{array}{l}1.0 \\
1.0\end{array}$ & $\begin{array}{l}0.0 \\
0.0\end{array}$ & $\begin{array}{l}0.0 \\
0.0\end{array}$ \\
\hline & $12 \mathrm{~m}$ & $\begin{array}{r}0 \\
100\end{array}$ & $\begin{array}{l}1.0 \\
1.0\end{array}$ & $\begin{array}{l}.142 \mathrm{E}-03 \\
.585 \mathrm{E}-04\end{array}$ & $\begin{array}{l}.389 \mathrm{E}-04 \\
.199 \mathrm{E}-06\end{array}$ \\
\hline & $4 \mathrm{~m}$ & $\begin{array}{r}0 \\
100\end{array}$ & $\begin{array}{l}.998 \\
.999\end{array}$ & $\begin{array}{l}.182 \mathrm{E}-(02 \\
.792 \mathrm{E}-(02\end{array}$ & $\begin{array}{l}.946 \mathrm{E}-04 \\
.588 \mathrm{E}-05\end{array}$ \\
\hline \multirow[t]{3}{*}{$\begin{array}{l}\text { Standard } \\
\text { Ditch }\end{array}$} & No object & $100 \%$ & $\begin{array}{l}.937 \\
.999\end{array}$ & $\begin{array}{l}.634 \mathrm{E}-01 \\
.117 \mathrm{E}-02\end{array}$ & $\begin{array}{l}.129 \mathrm{E}-(04 \\
.122 \mathrm{E}-(05\end{array}$ \\
\hline & $12 \mathrm{~m}$ & $\begin{array}{r}0 \\
100\end{array}$ & $\begin{array}{l}.936 \\
.999\end{array}$ & $\begin{array}{l}.640 \mathrm{E}-01 \\
.127 \mathrm{E}-02\end{array}$ & $\begin{array}{l}.196 \mathrm{E}-04 \\
.154 \mathrm{E}-05\end{array}$ \\
\hline & $4 \mathrm{~m}$ & $\begin{array}{r}0 \\
100\end{array}$ & $\begin{array}{l}.937 \\
.998\end{array}$ & $\begin{array}{l}.625 \mathrm{E}-01 \\
.181 \mathrm{E}-02\end{array}$ & $\begin{array}{l}.998 \mathrm{E}-04 \\
.658 \mathrm{E}-05\end{array}$ \\
\hline
\end{tabular}

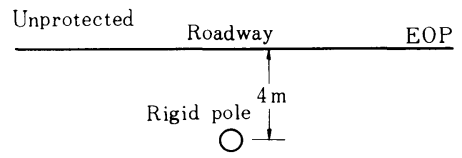

Short guardrail

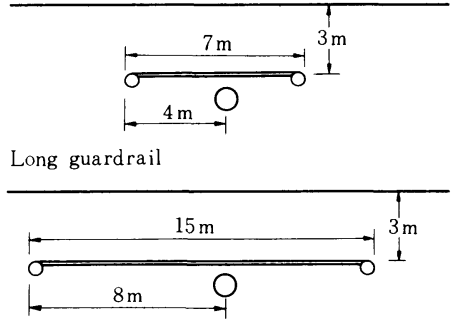

Fig. 4 Simulation Experiment (B) (Effect of guardrail protection)

Table 2 Simulation Result (B) (Effects of guardrail installation to protect a rigid pole)

\begin{tabular}{l|c|c|c|c}
\hline \multirow{2}{*}{$\begin{array}{c}\text { Guardrail } \\
\text { Length }\end{array}$} & \multirow{2}{*}{$\begin{array}{l}\text { Restraint } \\
\text { Usage }\end{array}$} & \multicolumn{3}{|c}{ Consequence probabilities } \\
\cline { 3 - 5 } & & ND/PDO & Injury & Fatal \\
\hline No guardrail & $0 \%$ & .937 & $.625 \mathrm{E}-01$ & $.998 \mathrm{E}-04$ \\
& 100 & .998 & $.181 \mathrm{E}-02$ & $.658 \mathrm{E}-05$ \\
Short guardrail & 0 & .941 & $.592 \mathrm{E}-01$ & $.633 \mathrm{E}-04$ \\
& 100 & .998 & $.187 \mathrm{E}-02$ & $.372 \mathrm{E}-05$ \\
Long guardrail & 0 & .947 & $.530 \mathrm{E}-01$ & $.105 \mathrm{E}-03$ \\
& 100 & .997 & $.263 \mathrm{E}-02$ & $.567 \mathrm{E}-05$ \\
\hline
\end{tabular}

\section{APPLICATIONS AND MODIFICATIONS OF RHSM}

The RHSM has been used in a number of studies and has undergone several revisions since it was first developed. Accompanying to the original study, a user's manual was developed to provide a simple procedure for estimating the degree of hazard represented by the roadside along any given section of highway without using a computer. The Roadside Hazard Probability Matrix has been created from a number of RHSM simulation runs to prepare a table which has all combinations of three types of terrain cross section geometries, three types of objects, two kinds of object rigidities and three object locations. The user can select the closest set of these variable combinations and estimate the consequence probabilities. The expected number of incidents of each type (no damage, PDO, injury and fatal) are calculated from the following equation.

$$
C_{i}=N \sum_{j}\left(L_{i} P_{i j}\right)
$$

where : $C_{i}=$ number of incidents of type $i$,

$N=$ number of encroachments per km per year,

$L_{i}=$ length of object component $j$ parallel to roadway, 
$P_{i j}=$ probability of incident type $i$ due to the presence of object component $j$

The number of encroachments per $\mathrm{km}$ per year must be obtained elsewhere and used as input to the estimation procedure. If the cost of each type of incident is defined, then the cost-effectiveness of a certain roadside hazard countermeasure can be tested. For example, shielding a rock-cut with a guardrail will decrease PDO, injury and fatal accidents at the cost of installing and maintaining the guardrail. The reduction in the cost of accidents will result in a net benefit if the encroachment rate is high enough.

The Road and Motor Vehicle Traffic Safety Branch, Transport Canada modified the computer program of the RHSM model for public distribution. They added a front-end routines to make the model more user friendly. This version (named as Version 5) accepts interactive input data entry and does preprocessing for the batch run. In addition, the cost-effectiveness analysis phase was incorporated as an integral part of the model.

Another extensive and substantial revision to the RHSM model was made in 1980 to study the effect of vehicle downsizing on the consequences of off-road excursions (DelCan 1981). The single vehicle trajectory routine assumed that no vehicle steer back occurred during an encroachment. A braking factor was used to account for cases where the wheels were locked and the vehicle slid down the roadside slope as opposed to the case where the vehicle wheels were rolling. An analysis of actual field data strongly suggested, however, that steer back is a very common occurrence and even the predominant situation. The revised model allows two choices. One is when the brakes are fully applied or wheels locked. The other is the brake is not applied and side slip is allowed depending on the slope and steering geometry.

Another modification is the assessment of vehicle stability on the slope transition point and on the down slope. The latter stability is associated with the steering angle and the side slip generated where a driver attempts a steer-back maneuver. As a rolled vehicle causes more serious consequences to occupants, the assessment of roll stability is an important model improvement (Cooper 1980).

The original RHSM assumed an intermediate size passenger car primarily due to the relationship with HVOSM. However, as the vehicle size is generally getting smaller and the effect of vehicle type difference is the subject of study, the revised RHSM model was extensively modified to incorporate eight different vehicle types. Not only the propensity of roll stability of different size, but the differences in impact consequences between large and small vehicles were examined and the model was modified. The revised RHSM was designated Version 6.

Twenty simulation runs were made with RHSM Version 6 covering 10 terrain and roadside object conditions for two vehicle sizes. The former were derived from a review of RTAC design standards together with knowledge of existing practice and are considered representative of a normal range of roadside conditions. The latter were the 1990 'design' mini-compact and larger cars respectively as assessed by the study group(Burtch et al. 1983).

The simulation results show that the occupants of small vehicles suffer higher probability of injury/fatality than do occupants of large vehicles when they crash with roadside objects such as poles and barriers. This is due to the shorter crash distance of small vehicles under otherwise identical crash conditions. The difference between consequences for small and large vehicles in the existing range of normal terrain negotiation is highly dependent on roll probability and the consequences of roll, and especially on the use of restraint systems. For various terrain conditions, the differences between large and small vehicles are relatively small if all the conditions are the same.

\section{ACKNOWLEDGEMENTS}

The author gratefully acknowledges financial support by the Road and Motor Vehicle Traffic Safety Branch, Transport Canada, and guidance and criticism by members of Roads and Trasportation Association of Canada Project Committee on Roadside Hazards during the model development. A special mention is due to his colleagues, Paul $\mathrm{O}$. Roer and Peter J. Cooper for their inspirations and valuable 


\section{suggestions.}

Last but not least the author wishes to express a sincere appreciation to the JSCE Committee for the invitation of the paper.

\section{REFERENCES}

1) American Association of State Highway and Transportation Officials : Guide for Selecting, Locating and Designing Traffic Barriers, 1977.

2) Burtch, T. M. et al : Vehicle Downsizing and Roadway Design in Canada, ITE Journal, February 1983.

3) Burgett. A. L. and Monk, M. W. : Car to Car Side Impacts : Computerized Crash Reconstruction. SAE Paper 751154, 1975.

4) Campbell, K. L. : Energy Basis for Collision Severity. SAE Paper 740565, 1974.

5) Cantilli, E. J. and Lee, B. : Treatment of Roadside Hazards - Decision and Design, HRR Special Report 107, pp 101-108, 1970 .

6) Cleveland, D. E. and Kitamura, R. : A Study of Two-Lane Rural Roadside Accidents, University of Michigan, 1976.

7) Cooper, P. J. : A Cost-benefit Model for Roadside Hazard Treatment, Symposium on Methods for Determining Geometric Road Design Standards, O. E. C. D., 1976.

8) Cooper, P. J. and Koike, H. : The Development of a Terrain Severity Model for Assessing the Need for Guardrail Placement on Embankments and the Use of this Model to Evaluate a Number of Different Cross-Section Configurations, prepared for the B. C. Ministry of Transportation, Communications and Highways, July 1980.

9) Cooper, P. J. and Koike, H. : Effect of Downsizing of Vehicles on Geometric Design of Cadian Highways- Technical Report on Modification of Roadside Hazard Simulation Model, prepared for Road and Motor Vehicle Traffic Safety Branch, Transport Canada, June 1981.

10) De Leuw Cather, Canada, Ltd. : The Relative Hazards of Roadside Obstacles and Design Elements, Vol. 1-4, 1975.

11) Dean, J. P. : Shoulder Design Standard and Highway Traffic Safety, University of New Brunswick, 1976.

12) DiLorenzo, F. A. : Power and Bodily Injury, SAE Paper 760014, 1976.

13) Dunlap, D. F. and Grote, P. : Median Dike Impact Evaluation: Sensitivity Analysis, HRR 386. pp. 111-122, 1972.

14) Edward, T. C. et al. : Development of Design Criteria for Safer Luminaire Supports, NCHRP Report 77.1969.

15) Gadd, C. W. : Use of a Weighted Impulse Criterion for Estimating Injury Hazard, SAE Paper 660793, 1966.

16) Glennon, J. C. : Priority Approach for Roadside Safety Improvements, Midwest Research Institute, 1973.

17) Glennon, J.C. : Roadside Safety Improvement Programs on Freeways: A Cost -Effectiveness Priority Approach, NCHRP Report 148, 1974.

18) Glennon, J. C. and Wilton, C. J. : Effectiveness of Roadside Safety Improvements : Vol 1. A Methodology of Determining the Sefety Effectiveness of Improvements on All Classes of Highways, Report No. FHWA-RD-75-23, 1974.

19) Glennon, J.C. and Tamburri, T. N. : Objective Criteria for Guardrail Installation, HRR 174, pp. 184 206, 1977.

20) Graf, N. L., et al. : Single Vehicle Accidents Involving Utility Poles, TRR 571, pp. 36 43, 1976.

21) Hall, J. W. : Identification and Programming of Roadside Hazard Improvements, University of Maryland, 1976.

22) Hirsh, T. J., et al : The Modular Crash Cushion-Research Findings and Field Experience, HRB Special Report 107, pp. 140 148, 1970 .

23) Hodgson, V. R., et al. : Testing the Validity and Limitations of the Severity Index, SAE Paper 700901, 1970.

24) Hutchinson, J. W. and Kennedy, T. W. : Medians of Divided Highways-Frequency and Nature of Vehicle Encroachments, Engineering Experiment Station Bulletin 487, University of Illinois, 1966.

25) Jones, I. S. and Segal, D. J. : The Application of the SMAC Accident Reconstruction Program to Actual Highway Accidents. Proceeding of 18 th AAAM Conference, 1974.

26) Jones, I. S. : The Effect of Vehicle Characteristics on Road Accidents, Pergamon Press, 1976.

27) Koike, H. : Roadside Hazard Simulation Model (RHSM), Interim Report, 1978.

28) Koike, H. and Roer, P. O. : Roadside Hazards-A Methodology and Techniques for Determining Accident Potentials, prepared for Transport Canada, 1978.

29) Koike, H. and Roer, P. O. : Roadside Hazard Simulation Model-User's Manual, prepared for Road and Motor Vehicle Safety Branch. Transport Canada, 1979.

30) Labra, J. J. et al. : Investigation of Canadian Concrete Guardrail Performance, Southwest Research Institute, 1977.

31) Langwieder, K. : Passenger Injuries in Collision and their Relation to General Speed Scale, SAE Paper $730963,1973$.

32) Marquis, E. L. et al. : Test and Evaluation of a Tire-Sand Inertia Barrier, TRR 556, pp. 69 79, 1976.

33) McHenry, R. R. et al. : Determination of Physicai Criteria for Roadside Energy Conversion Systems, Cornell Aeronautical Laboratory, CAL No. VJ-2251-V-1, 1967.

34) McHenry, R. R. and Deleys, N. J. : Vehicle Dynamics in Single Vehicle Accidents-Validation and Extensions of a Computer Simulation, Cornell Aeronautical Laboratory, CAL No. VJ-2252-V-3, 1968. 
35) McHenry, R. R. : A Computer Program for Reconstruction of Highway Accidents. Proceedings of the 17 th Stapp Car Crash Conference, 1973.

36) McHenry, R. R. : The CRASH Program-A Simplified Collision Reconstruction Program. Motor Vehicle Collision Investigation Symposium, Volume I, Proceedings, DOT HS-801979. 1976.

37) Melvin. J. W. et al. : Human Injury Mechanism and Impact Tolerance, TRR 586, pp. 11 22. 1976.

38) Michie, J. D. et al. : Guardrail Performance and Design, NCHRP Report 115, 1971.

39) Michie, J.D. et al. : Location, Selection and Maintenance of Highway Traffic Barriers, NCHRP Report 119, 1971

40) Michie, J. D. et al. : Guardrail Crash Test Evaluation: New Concept and End Designs, NCHRP Report $129,1972$.

41) Moffatt, C. A. : Computer Reconstruction and Accident Severity, Motor Vehicle Collision Investigation Symposium. Volume I : Proceedings, DOT HS-801979, 1976.

42) Newman, J. A. : On the Use of Head Injury Criterion (HIC) in Protective Headgear Evaluation, SAE Paper $751162,1975$.

43) Nowak, E. S. : Single Vehicle/Fixed Object Collision Study in Ontario Provincial Police District No. 2 for the Period January 1. 1976 to May 31. 1976. University of Western Ontario, Report SVFO-FR-1-76. 1976.

44) O. E. C. D. Road Research Group : Roadside Obstacles, 1975.

45) Olson, R. M. et al. : Bridge Rail Design - Factors. Trends and Guidelines, NCHRP Report 149, 1974.

46) Pinkney, H. F. L. et al. : The NAE Model of the Highway Cable Barrier for Parametric Studies of Vehicle Redirection Using Digital Simulation, Mechanical Engineering Report MS-133. National Research Council of Canada, 1972.

47) Ross, H. E. and Ivey. D. L. : Vehicle-Roadway Interaction Studies by Math Model, ASCE Transportation Engineering Journal, Vol. 98 N. TE 2, pp. 285 298, 1972.

48) Ross, H. E. and Post, E. R. : Tentative Criteria for the Design of Safe Sloping Culvert Grates, HRR 386, pp. 101 110, 1972.

49) Ross, H. E. and Post, E. R. : Warrants for Guardrails on Embankments, HRR 460, pp. 85〜96, 1973.

50) Ross, H. E. and Post, E. R. : Full-scale Embankment Tests and Comparison with a Computer Simulation, TRR 488, pp. 53 63. 1974.

51) Ross, H. E. : Impact Performance and an Evaluation Criterion for Median Barriers, TRR 586 pp. 32 49, 1976.

52) Roy Jorgensen and Associates, : Evaluation of Criteria for Safety Improvements on the Highway, 1976.

53) Rouze, W. S. and Gendre, F. : Field Experience and Evaluation of TAD Project Vehicle Damage Rating Scale, Proceedings of 13 th Stapp Car Crash Conference, 1969.

54) SAE Information Report J $885 \mathrm{a}$, : Human Tolerance to Impact Conditions as Related to Motor Vehicle Design, 1966.

55) SAE Information Report J 224 a, : Collision Deformation Classification, 1975.

56) Segal, D. J. : Highway-Vehicle-Object Simulation Model-1976, Vol. 1-4, Report No. FHWA-RD-76-162 through $165,1976$.

57) Tarriere, C. et al. : The Contribution of Physical Analysis of Accidents Towards Interpretation of Severe Traffic Trauma, SAE Paper 751176, 1975.

58) Transportation Centre, : Study of the Relationship Between Vehicle Impact Speed and Various Severity Parameters, University of Saskachewan, 1976.

59) Tutt, P. R. and Nixon, J. F. : Roadside Design Guidelines, HRB Special Report 107, pp. 119 132, 1970.

60) Versace, J. : A Review of the Severity Index, SAE Paper 710881, 1971.

61) Walton, N. E. et al. : Evaluation of Breakaway Light Poles for Use in Highway Medians, HRR 460, pp. 123 136, 1973.

62) Watson, L. G. and Shiels, A. C. : Injury Predictions for Frontal Collisions, SAE Paper 751171, 1975.

63) Weaver, G. D. et al. : Selection of Safe Roadside Cross Sections, NCHRP Report 158, 1975.

64) Wright, P. H. and Robertson, L. S. : Priorities for Roadside Hazard Modification : A Study of 300 Fatal Roadside Object Crashes, Insurance Institute of Highway Safety. 1976.

65) Young, R. D. et al. : Simulation of Vehicle Impact with Texas Concrete Median Barrier: Test Comparisons and Parameter Study, HRR 460, pp. 61 72, 1973.

(Received April 22 1985) 